Cita: Gomà-i-Freixanet, M.; Pla-Cortés, J.; Avilés-Antón, O. (2020). Perfil diferencial de personalidad de los árbitros de élite del baloncesto español. Cuadernos de Psicología del Deporte, 20(1), 1-9

\title{
Perfil diferencial de personalidad de los árbitros de élite del baloncesto español
}

\section{Differential personality profile of elite Spanish basketball referees}

\section{Perfil diferencial de personalidade dos árbitros de elite do basquetebol espanhol}

\author{
Gomà-i-Freixanet, M.; Pla-Cortés, J.; Avilés-Antón, O.
}

\section{Departamento de Psicología Clínica y de la Salud, Facultad de Psicología, Universitat Autònoma de} Barcelona, Barcelona, España.

\section{RESUMEN}

El objetivo del presente estudio consistió en averiguar si existe un perfil diferencial de personalidad de los árbitros de baloncesto españoles según el nivel de ejecución alcanzado. Administramos el Inventario de Personalidad NEO Revisado (NEO PI-R; Costa y McCrae, 1999) a 418 árbitros varones asignándolos a dos categorías: grupo de Éxito (pertenecientes a las ligas ACB y LEB) y grupo de No-Éxito (pertenecientes a las competiciones del Comité de Árbitros de la Federación Catalana de Baloncesto). Durante cinco temporadas hicimos un seguimiento para cerciorarnos que los árbitros del grupo de NoÉxito no ascendían en su carrera deportiva y asegurar que las categorías eran estancas. La comparación de ambos grupos nos indica que los árbitros que alcanzan la élite son personas con una mayor competencia y asertividad, seguras de sí mismas, eficientes y organizadas, así como dominantes y con dotes de liderazgo. Sugerimos que los responsables de organizar y dirigir la formación de los árbitros deportivos deberían incluir en sus programas actividades que fomentaran las habilidades comunicativas y de liderazgo propias de aquellos que alcanzan la élite.

Palabras clave: Psicología del deporte; personalidad; NEO PI-R; arbitraje deportivo.

\section{ABSTRACT}

The aim of the present study was to find out if there is a differential personality profile at the Spanish basketball referees according to their performance level. We administered the NEO Revised Personality Inventory (NEO PI-R; Costa \& McCrae, 1999) to 418 male referees by assigning them to two categories: the Successful Group (belonging to the ACB and LEB league competitions) and the Unsuccessful Group (belonging to the competitions of the regional Catalan Basketball Federation). After five seasons, we contacted again the referees of the unsuccessful group to make sure that they did not promote in their sports career and to ensure that the categories were not permeable. The comparison of both groups indicates that the referees who reach the elite display greater competence and assertiveness, are self- 


\section{Gomà-i-Freixanet, Pla-Cortés, Avilés-Antón.}

assured, efficient and organized, as well as dominant and with leadership skills. We suggest that those leading and organizing the training of sports referees should include in their programs activities promoting the skills -leadership and communication- of those who have already reached the elite.

Keywords: Sports psychology; personality; NEO Personality Inventory; sports officiating.

\section{RESUMO}

O objectivo do presente estudo foi averiguar se existe um perfil diferencial de personalidade dos árbitros de basquetebol espanhol de acordo com o nível de execução alcançado. Realizámos o questionário NEO Revised Personality Inventory (NEO PI-R; Costa e McCrae, 1999) a 418 árbitros masculinos, atribuindoos a duas categorias: o grupo Sucesso (pertencente às competições das Ligas ACB e LEB) e o grupo nãoSucesso (pertencente às competições do Comité de Árbitros da Federação Catalã de Basquetebol). Durante cinco temporadas realizou-se um seguimento para garantir que os árbitros do grupo Não-Sucesso não ascendiam na sua carreira desportiva e assegurar que as categorias fossem estáveis. Uma análise comparativa de ambos os grupos indica que os árbitros que alcançam a elite são pessoas com maior competência e assertividade, autoconfiança, eficiencia e organização, bem como pessoas dominantes e com habilidades de liderança. Desta forma, sugerimos, que os responsáveis pela organização e direcção do treino de árbitros desportivos deveriam incluir nos seus programas actividades que promovessem habilidades comunicativas e de liderança, proprias daqueles que alcançam a elite.

Palavras chave: Psicologia no desporto; personalidade; NEO PI-R; arbitragem desportiva.

\section{INTRODUCCIÓN}

A pesar de la importancia de sus decisiones, la psicología del deporte ha prestado poca atención a los árbitros y jueces deportivos. Uno de los temas tradicionalmente estudiados en este ámbito ha sido las fuentes de estrés que inciden en la labor arbitral y la manera de afrontarlo (véase por ejemplo Hill, Matthews, y Senior, 2016; Johansen y Haugen, 2013; Martin, 2009). Últimamente, los estudios se han focalizado en los aspectos que influyen en las decisiones arbitrales, tales como la presión del público (Di Corrado, Pellarin, y Agostini, 2011; Myers y Balmer, 2012; Unkelbach y Memmert, 2010), el proceso de toma de decisiones (Burnett, Bishop, Ashford, Williams, y Kinrade, 2017; Neville, Salmon y Read, 2017; Souchon et al., 2016) o la autoeficacia arbitral (Lirgg, Feltz, y Merrie, 2016; Myers, Feltz, Guillén, y Dithurbide, 2012).

Sin embargo, apenas hay investigaciones acerca de la personalidad de los agentes que toman estas decisiones, es decir, de los propios árbitros. La revisión de los pocos estudios publicados en esta materia nos indica que los resultados han sido poco concluyentes probablemente debido a las diferentes metodologías utilizadas y a los distintos instrumentos de evaluación. Así, en la década de los años 1970-80, para la evaluación de la personalidad, los estudios (véase por ejemplo, Alker, Straub, y Leary, 1973; Dale, 1976; Ittenbach y Eller, 1988; Sinclair, 1975) utilizaron mayoritariamente el Inventario Psicológico de California (CPI; Gough, 1964) y en menor cuantía (véase por ejemplo, Spurgeon, Blair, Keith, y McGinn, 1978) el Cuestionario Factorial de Personalidad (16 PF; Cattell, 1972). Los estudios acabados de mencionar indicarían que la Dominancia (CPI) o alguna de sus manifestaciones, tales como la Autosuficiencia o el Liderazgo, serían características definitorias de los árbitros de mayor nivel o experiencia profesional, y que los árbitros de fútbol americano con mayor experiencia comparados con los que están a prueba tenderían a puntuar más alto en Extraversión (16 PF).

Más recientemente, se han publicado estudios que han utilizado los cuestionarios desarrollados por Costa y McCrae (NEO PI-R y NEO-FFI; Costa y McCrae, 1992). Así, por ejemplo, Balch y Scott (2007) hallaron que los árbitros de voleibol, hockey y lucha puntuaban significativamente más alto que la muestra normativa en Extraversión (NEO-FFI); mientras que Winters (2010) concluyó que los árbitros de béisbol de una competición superior universitaria en comparación con los árbitros de competiciones inferiores mostraban niveles moderados de Fantasía y Modestia, y bajos de 


\section{Personalidad y arbitraje de élite del baloncesto español}

Gregarismo (NEO PI-R). Otros autores (Pla-Cortés, Gomà-i-Freixanet, y Avilés-Antón, 2015), utilizando el NEO PI-R y comparando los árbitros de baloncesto españoles con las puntuaciones normativas poblacionales, encontraron que los árbitros obtenían puntuaciones significativamente más altas en Neuroticismo, y más bajas en Apertura a la Experiencia, Amabilidad y Responsabilidad. Así pues, a partir de la revisión de la literatura acerca del perfil de personalidad de los árbitros evaluado mediante el CPI o el 16PF podemos concluir que cuando se comparan los árbitros, bien sean aspirantes o de categorías inferiores con aquellos que dirigen partidos de la élite profesional, las variables que se relacionan con la excelencia en el desempeño de la labor arbitral son la Dominancia y la Extraversión. Sin embargo, los resultados obtenidos a partir del NEO no permiten establecer conclusiones fehacientes ya que los estudios no son comparables entre sí.

Por otra parte, y como hemos mencionado anteriormente, debemos destacar que en el estudio del perfil de personalidad de los árbitros y jueces deportivos nos encontramos ante una consideración de tipo metodológico. Así, observamos que los estudios que comparan los árbitros de categorías superiores con los de categorías inferiores no toman en consideración que todos los árbitros empiezan arbitrando en las categorías de base, y que a lo largo de su práctica deportiva mejoran sus ejecuciones y progresan en su carrera arbitral, pudiendo llegar a la élite. A este fenómeno lo hemos denominado puerta abierta, ya que la composición no estanca de ambas categorías dificulta la interpretación de los resultados obtenidos.

A partir de la revisión de la literatura en el ámbito de la personalidad y del arbitraje deportivo, y de los problemas metodológicos descritos, el objetivo del presente estudio consiste en describir el perfil de personalidad de una muestra representativa de árbitros de la élite del baloncesto español que denominamos Grupo de Éxito y compararlo con una muestra de árbitros que nunca alcanzó las categorías altas y que denominamos Grupo de No-Éxito. A diferencia de los estudios mencionados anteriormente, la selección del Grupo de No-Éxito se realizó siguiendo unos criterios de inclusión según los cuales las posibilidades de alcanzar las máximas categorías eran prácticamente nulas. Asimismo, durante cinco temporadas hicimos un seguimiento de este grupo para asegurarnos que no habían progresado y que se mantenían en las categorías bajas. Dicha estrategia es una aportación importante de nuestro trabajo, ya que, según nuestro conocimiento, esta es la primera vez que se lleva a cabo un estudio con árbitros que cumplen criterios de impermeabilidad entre categorías.

A partir de la literatura consultada, hipotetizaríamos que los árbitros pertenecientes al Grupo de Éxito puntuarían más alto en Extraversión y Dominancia que los árbitros pertenecientes al Grupo de No-Éxito. Sin embargo, en nuestro estudio, al utilizar una metodología diferente para la categorización de los grupos a comparar, hipotetizaríamos además que el Grupo de Éxito mostrará unas características diferenciales de personalidad que le hará más apto para el arbitraje, tales como las relacionadas con el liderazgo y la autoconfianza en el terreno de juego.

\section{MATERIAL Y MÉTODOS}

\section{Participantes}

La muestra inicial estuvo compuesta por 418 árbitros varones que militaban en todas las categorías del baloncesto español, desde las más altas a las de base. Se excluyeron aquellos participantes que no cumplían los criterios de validez del NEO PI-R $(n=10 ; 2,39 \%)$ $\mathrm{y}$ aquellos que omitieron algún dato en el cuestionario sociodemográfico $(n=6 ; 1,43 \%)$. Finalmente, también se excluyeron aquellos que, tras el seguimiento de las cinco temporadas deportivas, no cumplían los criterios de inclusión para pertenecer a una de las dos categorías estudiadas: Éxito vs. NoÉxito $(n=255 ; 61 \%)$.

La muestra total final quedó constituida por 147 árbitros de baloncesto $(M=29,38$ años; $S D=5,58$; rango $=20-47)$. El Grupo de Éxito $(n=73)$ estuvo compuesto por los árbitros que habían conseguido las máximas categorías del baloncesto español, y el Grupo de No-Éxito $(n=74)$ por el resto de miembros que cumplían los criterios para pertenecer a dicha categoría (Figura 1). 


\section{Gomà-i-Freixanet, Pla-Cortés, Avilés-Antón.}

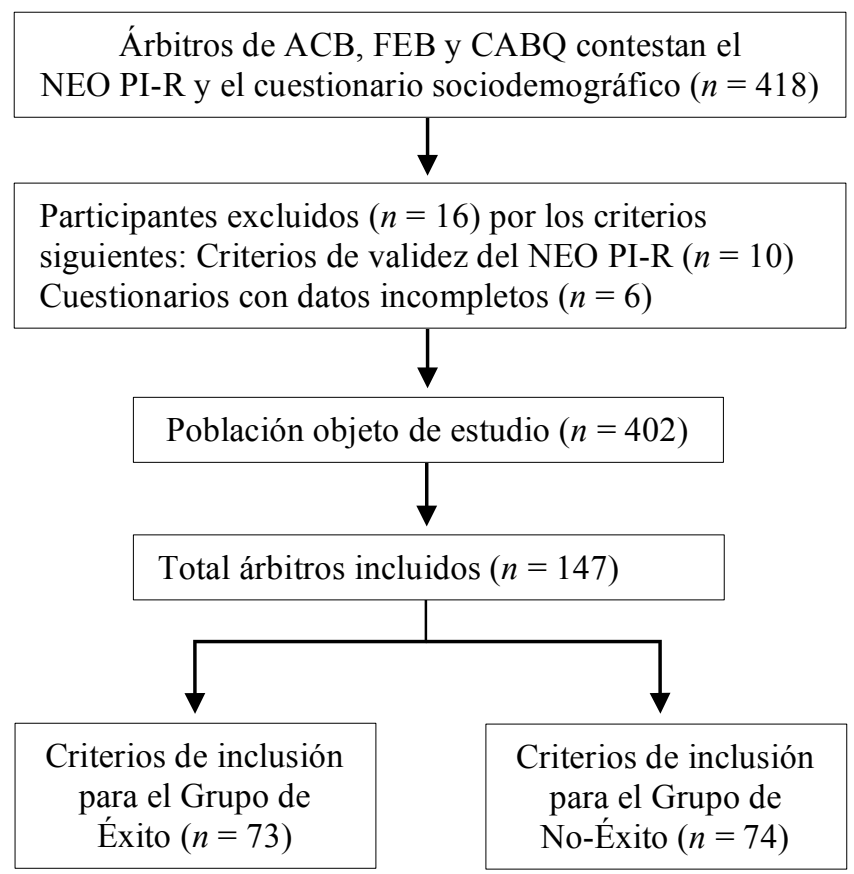

Figura 1. Diagrama de flujo de los criterios de exclusión e inclusión de la muestra de estudio.

Nota. $A C B=$ Asociación de Clubes de Baloncesto;

$F E B=$ Federación Española de Baloncesto;

$C A B Q=$ Comité de Árbitros de la Federación Catalana de Baloncesto; NEO PI-R = Inventario de Personalidad NEO Revisado.

\section{Instrumentos}

Para la evaluación de la personalidad administramos la versión española del Inventario de Personalidad NEO Revisado (NEO PI-R; Costa y McCrae, 1999), forma S (auto-informe). El cuestionario consta de 240 elementos que se contestan en una escala Likert de cinco opciones que va del totalmente de acuerdo (1) al totalmente en desacuerdo (5). Dicho test evalúa cinco dominios de personalidad: Neuroticismo $(\mathrm{N})$, Extraversión (E), Apertura a la Experiencia (O), Amabilidad (A) y Responsabilidad (C), y cada uno de los dominios evalúa a su vez seis facetas.

La elección de dicho modelo de personalidad se basa, aparte de su robustez conceptual, en que nos permite un análisis muy pormenorizado del perfil diferencial de los árbitros al proporcionarnos un total de 35 puntuaciones diferentes.

\section{Procedimiento}

Administramos el NEO PI-R y un cuestionario demográfico diseñado para este estudio a un grupo representativo de árbitros españoles de baloncesto de todas las categorías. Una vez transcurridas las cinco temporadas deportivas desde la evaluación, los participantes fueron asignados a dos grupos: Grupo de Éxito, compuesto por aquellos árbitros que habían alcanzado las máximas categorías del baloncesto español; y Grupo de No-Éxito, compuesto por aquellos árbitros cuya posibilidad de alcanzar las máximas categorías era nula.

Los criterios de inclusión al Grupo de Éxito fueron: pertenecer a la máxima categoría del baloncesto español denominada Liga de la Asociación de Clubes de Baloncesto (Liga ACB), o a la categoría inmediatamente inferior a ésta, la Liga Española de Baloncesto (Liga LEB), que es la máxima competición organizada por la Federación Española de Baloncesto (FEB). Hemos considerado que los árbitros que llegan a una de estas dos categorías han alcanzado la excelencia en su carrera deportiva ya que los jugadores de las mismas son profesionales.

Por otra parte, los criterios de inclusión para pertenecer al Grupo de No-Éxito fueron haber empezado a arbitrar con una edad $\leq 18$ años y cumplir uno de los dos criterios: a) No haber alcanzado la Liga Española de Baloncesto Amateur (Liga EBA, la primera de las competiciones regulares de la FEB) a lo largo de 11 años de carrera arbitral; o b) Ser árbitro del Comité de Árbitros de la Federación Catalana de Baloncesto (CABQ) y haber descendido de cualquier categoría a lo largo de su carrera arbitral.

Para determinar los criterios de inclusión al Grupo de No-Éxito, establecimos como principio que sus miembros estuvieran totalmente estancados en su carrera arbitral, bien porque llevaran demasiado tiempo arbitrando en categorías inferiores o bien porque hubieran descendido de una categoría superior en la que estuviera arbitrando. Ambas situaciones serían indicadoras de escasas aptitudes arbitrales. Así pues, consideramos que quienes hubieran descendido de categoría o no hubieran alcanzado la Liga EBA tras 11 años de carrera en el $\mathrm{CABQ}$, difícilmente lo conseguirían. Dicho criterio temporal de 11 años lo establecimos a partir de la 


\section{Personalidad y arbitraje de élite del baloncesto español}

media en temporadas que los árbitros pertenecientes al Grupo de Éxito tardaron en alcanzar dicha categoría. Por otro lado, el criterio de establecer un tope en la edad de inicio en el arbitraje nos aseguraba que los árbitros no tenían más limitaciones que las técnicas o personales ya que se considera que un inicio tardío en el arbitraje limita las posibilidades de proyección deportiva. Dicho criterio temporal lo establecimos también a partir de la media de edad de inicio en el arbitraje de los árbitros de la Liga ACB de nuestra muestra.

Para la realización de dicho estudio obtuvimos la aprobación del Comité y de la Escuela de Árbitros de la Federación Catalana de Baloncesto y de la Federación Española. Al ser un estudio anónimo, los participantes recibieron un código alfanumérico que permitía hacer el seguimiento transcurridas las cinco temporadas deportivas. Ambos cuestionarios fueron administrados en grupo durante las concentraciones de inicio de temporada. La participación fue voluntaria $y$ todos firmaron el consentimiento informado. El estudio se realizó siguiendo las directrices de la Declaración de Helsinki (World Medical Association, 2013).

\section{Análisis estadístico}

Para la comparación de ambos grupos utilizamos un ANCOVA controlando el efecto de la edad y calculamos el tamaño del efecto mediante la $d$ de Cohen. Analizamos los datos recogidos mediante el programa estadístico Statistical Package for the Social Sciences, versión 20.

\section{RESULTADOS}

La muestra final quedó constituida por 147 árbitros: Grupo de Éxito $(M=32,60$ años; $S D=5,01$; rango $=$ $25-47)$ y Grupo de No-Éxito $(M=26,20 ; S D=4,12$; rango $=20-39)$. La Tabla 1 muestra la distribución de los participantes según el grupo de pertenencia, el nivel de logro alcanzado y la categoría arbitral.

Ambos grupos se diferenciaron significativamente en edad $(F=71,62 ; p>, 001)$. En relación a las variables de personalidad del NEO PI-R (véase Tabla 2), ambos grupos no se diferenciaron significativamente en ninguno de los dominios. Sin embargo, sí se hallaron diferencias significativas en la faceta Competencia (C1) con un tamaño del efecto considerado de magnitud media.

Tabla 1. Número de participantes y estadísticos descriptivos de la variable edad, en función del nivel de logro alcanzado y la categoría arbitral.

\begin{tabular}{|c|c|c|c|}
\hline \multirow[t]{2}{*}{ Grupo y categoría } & \multicolumn{3}{|c|}{ Edad (años) } \\
\hline & $n$ & $M$ & $S D$ \\
\hline \multicolumn{4}{|l|}{ Grupo de Éxito } \\
\hline $\mathrm{ACB}$ & 38 & 34,87 & 5,49 \\
\hline LEB & 35 & 30,14 & 2,89 \\
\hline Total árbitros & 73 & 32,60 & 5,01 \\
\hline \multicolumn{4}{|l|}{ Grupo de No-Éxito } \\
\hline Diversas categorías del CABQ & 74 & 26,20 & 4,12 \\
\hline TOTAL & 147 & 29,38 & 5,58 \\
\hline
\end{tabular}

Nota. $A C B=$ Asociación de Clubes de Baloncesto; $L E B=$ Liga Española de Baloncesto; $C A B Q=$ Comité de Árbitros de la Federación Catalana de Baloncesto. 


\section{Gomà-i-Freixanet, Pla-Cortés, Avilés-Antón.}

Tabla 2. Análisis de covarianza de las dimensiones y facetas del NEO PI-R, entre los grupos de árbitros de éxito y de no-éxito controlando la edad, y tamaño del efecto.

\begin{tabular}{|c|c|c|c|c|c|c|c|}
\hline & \multicolumn{2}{|c|}{$\begin{array}{l}\text { Grupo de Éxito } \\
\qquad(n=73)\end{array}$} & \multicolumn{2}{|c|}{$\begin{array}{c}\text { Grupo de } \\
\text { No-Éxito } \\
(n=74)\end{array}$} & \multirow[b]{2}{*}{$\boldsymbol{F}$} & \multirow[b]{2}{*}{$p$} & \multirow[b]{2}{*}{$\begin{array}{c}d \text { de } \\
\text { Cohen }\end{array}$} \\
\hline & $\boldsymbol{M}$ & $S D$ & $\boldsymbol{M}$ & $S D$ & & & \\
\hline Edad (años) & 32,60 & 5,01 & 26,20 & 4,12 & 71,62 &, 001 & 1,40 \\
\hline \multicolumn{8}{|l|}{ NEO PI-R } \\
\hline Neuroticismo (N) & 70,12 & 18,34 & 75,27 & 20,16 & 1,65 & 201 & 0,27 \\
\hline Extraversión $(\mathrm{E})$ & 113,11 & 18,24 & 114,64 & 22,42 & 0,87 &, 352 & 0,07 \\
\hline Apertura a la Experiencia (O) & 104,21 & 20,17 & 107,80 & 19,74 & 0,50 & ,480 & 0,18 \\
\hline Amabilidad(A) & 121,73 & 16,76 & 115,50 & 16,22 & 0,91 & ,343 & 0,38 \\
\hline Responsabilidad (C) & 130,92 & 17,81 & 122,15 & 24,35 & 1,92 &, 167 & 0,41 \\
\hline Ansiedad (N1) & 14,37 & 4,62 & 15,30 & 4,17 & 1,03 &, 310 & 0,21 \\
\hline Hostilidad (N2) & 9,88 & 4,52 & 10,81 & 4,90 & 1,67 & ,198 & 0,20 \\
\hline Depresión (N3) & 10,27 & 4,24 & 11,91 & 5,18 & 3,45 &, 065 & 0,35 \\
\hline Ansiedad Social (N4) & 12,68 & 4,25 & 12,96 & 5,08 & 0,01 & ,932 & 0,06 \\
\hline Impulsividad (N5) & 14,56 & 3,99 & 15,41 & 4,56 & 0,43 &, 515 & 0,20 \\
\hline Vulnerabilidad (N6) & 8,36 & 3,75 & 8,89 & 4,07 & 0,32 &, 572 & 0,14 \\
\hline Cordialidad (E1) & 21,42 & 4,31 & 21,59 & 4,48 & 0,44 &, 510 & 0,04 \\
\hline Gregarismo (E2) & 16,90 & 5,22 & 17,61 & 6,44 & 0,34 &, 558 & 0,12 \\
\hline Asertividad (E3) & 19,14 & 4,22 & 17,95 & 5,24 & 3,87 &, 051 & 0,25 \\
\hline Actividad (E4) & 19,38 & 3,97 & 19,62 & 4,74 & 0,08 & ,772 & 0,05 \\
\hline Búsqueda de emociones (E5) & 14,62 & 4,34 & 15,88 & 4,84 & 0,00 & ,969 & 0,27 \\
\hline Emociones positivas (E6) & 21,64 & 4,92 & 21,99 & 5,94 & 0,73 & ,393 & 0,06 \\
\hline Fantasía (O1) & 16,33 & 5,16 & 17,95 & 5,17 & 2,69 &, 103 & 0,31 \\
\hline Estética $(\mathrm{O} 2)$ & 16,04 & 5,23 & 15,30 & 6,06 & 0,20 & ,652 & 0,13 \\
\hline Sentimientos (O3) & 19,85 & 4,31 & 19,15 & 4,13 & 1,45 &, 230 & 0,17 \\
\hline Acciones $(\mathrm{O} 4)$ & 15,00 & 4,28 & 15,74 & 4,44 & 0,09 & ,761 & 0,17 \\
\hline Ideas (O5) & 16,10 & 5,69 & 17,53 & 5,83 & 2,50 &, 116 & 0,25 \\
\hline Valores (O6) & 20,89 & 4,05 & 22,14 & 3,72 & 2,01 & , 158 & 0,32 \\
\hline Confianza (A1) & 21,49 & 4,35 & 19,19 & 5,83 & 3,22 & 074 & 0,45 \\
\hline Franqueza (A2) & 18,32 & 4,50 & 16,35 & 5,20 & 0,62 & ,431 & 0,41 \\
\hline Altruismo (A3) & 22,04 & 4,40 & 22,16 & 3,71 & 0,18 & ,673 & 0,03 \\
\hline Actitud conciliadora (A4) & 17,47 & 4,41 & 17,47 & 4,36 & 0,43 &, 513 & 0,00 \\
\hline Modestia (A5) & 19,96 & 4,15 & 18,92 & 4,34 & 0,86 &, 354 & 0,24 \\
\hline Sensibilidad a los demás (A6) & 22,45 & 3,71 & 21,41 & 2,98 & 0,01 & ,940 & 0,31 \\
\hline Competencia $(\mathrm{C} 1)$ & 22,95 & 3,46 & 20,73 & 4,64 & 6,78 &, 010 & 0,54 \\
\hline Orden $(\mathrm{C} 2)$ & 19,38 & 5,05 & 17,92 & 5,59 & 0,04 & ,837 & 0,27 \\
\hline Sentido del deber (C3) & 24,38 & 3,24 & 22,81 & 4,77 & 1,44 &, 231 & 0,39 \\
\hline Necesidad de logro (C4) & 22,73 & 3,70 & 21,47 & 4,71 & 0,81 &, 370 & 0,30 \\
\hline Autodisciplina (C5) & 21,66 & 4,55 & 21,50 & 5,38 & 0,05 &, 818 & 0,03 \\
\hline Deliberación (C6) & 19,82 & 4,43 & 17,72 & 6,39 & 3,45 &, 065 & 0,38 \\
\hline
\end{tabular}




\section{Personalidad y arbitraje de élite del baloncesto español}

\section{DISCUSIÓN}

Los resultados de nuestro estudio indican que ambos grupos de árbitros no se diferencian significativamente en ninguno de los dominios de personalidad evaluados por el NEO PI-R. Sin embargo, sí que hallamos diferencias significativas en la faceta Competencia indicando que el perfil de personalidad de los árbitros que alcanzan el éxito, en comparación con los que no lo alcanzan nos indica que son personas que se sienten más preparadas, más seguras de sí mismas, eficientes y organizadas, así como dominantes y con dotes de liderazgo. Así, puntuaciones altas en dicha variable, que es una faceta del dominio que evalúa Responsabilidad, nos indicarían a su vez un tipo de persona prudente, eficaz, bien preparada y que confía en sus propias capacidades. Estas características de personalidad son cualidades que un árbitro de la máxima categoría debe poseer para lograr un excelente desempeño en su labor. Dicha confianza en sí mismo le capacita no solo para ser eficaz en su tarea, sino para soportar las críticas acerca de sus decisiones arbitrales tanto en el campo por parte de los jugadores y público, como por parte de los medios de comunicación una vez ha finalizado el partido. En definitiva, puntuaciones elevadas en Competencia indicarían que son personas que asumen sus propias decisiones arbitrales, tanto los aciertos como los errores. Dichos resultados van en la misma dirección que los obtenidos por Alker et al. (1973), Dale (1976) e Ittenbach y Eller (1988) utilizando el CPI, en el sentido de una mayor auto aceptación en los árbitros de mayor nivel o experiencia profesional.

Cabe destacar que la variable Asertividad, que es una faceta del dominio que evalúa Extraversión, llega casi a la significación estadística, siendo el grupo de éxito el que obtuvo puntaciones más elevadas. Esta mayor Asertividad de los árbitros de élite indicaría una mejor capacidad para desenvolverse adecuadamente en los conflictos interpersonales que puedan surgir en el terreno de juego. En la alta competición, las habilidades que poseen las personas asertivas como la persuasión, el tacto y una comunicación eficaz pueden ser determinantes para manejar situaciones conflictivas haciendo más eficaz la actividad arbitral. Pero la faceta Asertividad del NEO PI-R evalúa también la dominancia, rasgo que hallaron elevado estudios anteriores (Alker et al.,
1973; Dale 1976; Fratzke, 1975), y que describieron una mayor dominancia en árbitros que dirigían competiciones de mayor nivel. En este mismo sentido, nuestros resultados avalan los obtenidos por Sinclair (1975), quien concluyó que los árbitros de mayor experiencia poseían una mayor capacidad de liderazgo y de persuasión. Aunque experiencia y éxito arbitral no son estrictamente equiparables, ya que en el grupo de árbitros que no han alcanzado el éxito los hay con mucha experiencia, sí que es cierto que los que alcanzan la élite lo hacen después de muchos años de dedicación y esfuerzo.

Finalmente, señalar que las facetas Confianza, Deliberación y Depresión también se aproximaron a la significación estadística con unos tamaños del efecto próximos a una magnitud media. Los árbitros de élite puntuaron más alto en confianza y deliberación, y más bajo en depresión, indicando que no se desaniman fácilmente, y que son prudentes a la hora de tomar decisiones transcendentes para el desarrollo del juego. Futuras investigaciones sobre el perfil de personalidad de dichos deportistas pueden arrojar luz sobre la importancia de dichas variables.

A tenor de los resultados obtenidos, podemos concluir que los árbitros que han alcanzado los máximos niveles competitivos se caracterizarían por tener una alta autoestima, ser más eficaces, asertivos, dominantes y con dotes de liderazgo, todo lo cual redunda en un mejor desempeño de la labor arbitral, y por ende facilita alcanzar la élite. Sin embargo, cabe destacar que la dominancia no debería entenderse como autoritarismo. Si bien es cierto que el perfil prototípico del árbitro de antaño era el de una persona punitiva, dominante, inflexible y autoritaria, en la actualidad se valora su tono conciliador, empático y con buena capacidad para la comunicación. Dichas características de personalidad proporcionan a los partidos el ritmo necesario para que se conserve el espectáculo deportivo y no se entre en una espiral de comportamientos antideportivos, sin olvidar que la dominancia extrema entra en conflicto con la vertiente pedagógica que exige el rol arbitral (Dosil, 2003; Guardo y Fleitas, 2004).

En relación a las limitaciones, cabe señalar que nuestro estudio adolece de una muestra de árbitros del género femenino, dada la escasa representación que había de mujeres árbitros, sobre todo en la élite. 


\section{Gomà-i-Freixanet, Pla-Cortés, Avilés-Antón.}

Esperemos que en un futuro se incremente la representación de dicho género en los niveles más altos del arbitraje de baloncesto español y que en estudios posteriores puedan realizarse las comparaciones oportunas para validar los resultados obtenidos en muestras masculinas.

Finalmente señalar que la incorporación de la evaluación de la personalidad en los estudios del ámbito del deporte en general aporta un valor añadido, ya que en el desempeño deportivo, no solo importa el "saber hacer" sino también el "saber estar".

\section{APLICACIONES PRÁCTICAS}

A modo de conclusión, consideramos que los responsables de dirigir y organizar los planes de formación de los árbitros, independientemente de la disciplina a la cual pertenezcan, les demandan que exhiban una serie de competencias que no siempre ostentan. Así pues, para implementarlas en su práctica diaria sería muy deseable incluir en los programas formativos actividades que habilitaran en cuantas competencias psicológicas se requieran para el óptimo arbitraje. Dicha formación ya ha sido demandada por los propios árbitros (Guillén, Morán, y Castro, 1999), la cual les permitiría incrementar y mejorar sus habilidades a la vez que proyectarse a la elite en sus respectivas disciplinas.

\section{AGRADECIMIENTOS}

En memoria del profesor Félix Guillén, maestro y compañero. Investigó, como pocos y con pasión, el mundo del arbitraje y del juicio deportivo. Te echamos de menos.

\section{REFERENCIAS}

1. Alker, H. A., Straub, W. F. y Leary, J. (1973). Achieving consistency: A study of basketball officiating. Journal of Vocational Behavior, 3, 335-343.

2. Balch, M. J. y Scott, D. (2007). Contrary to Popular Belief, Refs are People Too! Personality and Perceptions of Officials. Journal of Sport Behavior, 30, 3-20.

3. Burnett, A. M., Bishop, D. T., Ashford, K. J., Williams, A. M. y Kinrade, N. P. (2017).
Decision-making of English Netball Superleague umpires: Contextual and dispositional influences. Psychology of Sport and Exercise, 31, 52-60. doi:10.1016/j.psychsport.2017.04.003

4. Cattell, R. B. (1972). Manual for the 16 PF (Sixteen Personality Factors). Illinois (USA): Institute for Personality and Ability Testing.

5. Costa, P. T. y McCrae, R.R. (1992). Revised NEO Personality Inventory (NEO PI-R) and the NEO Five-Factor Inventory (NEO-FFI) professional manual. Odessa, FL: Psychological Assessment Resources.

6. Costa, P. T. y McCrae, R. R. (1999). Inventario de personalidad NEO Revisado (NEO PI-R). Inventario NEO Revisado de Cinco Factores (NEO-FFI). Manual profesional. Madrid: TEA Ediciones, S.A.

7. Dale, J. P. (1976). An investigation of personality characteristics of baseball umpires (Trabajo de máster no publicado). California State University, Pomona. CA.

8. Di Corrado, D., Pellarin, E. y Agostini, T. A. (2011). The phenomenon of social influence on the football pitch: Social pressure from the crowd on referees' decisions. Review of Psychology, 18(1), 33-36.

9. Dosil, J. (2003). La función educativa del árbitro y juez deportivo. En F. Guillén (Director), Psicología del arbitraje y el juicio deportivo (pp. 133-160). Barcelona: Inde.

10. Fratzke, M. R. (1975). Personality and biographical traits of superior and average college basketball officials. The Research Quarterly, 46, 484-488.

11. Gough, H. G. (1964). The California psychological inventory. Palo Alto: Consulting Psychologists Press.

12. Guardo, M. E. y Fleitas, C. I. (2004). Hacia una teoría del arbitraje deportivo: introducción. Lecturas: Educación Física y deportes, 68. Extraído de: http://www.efdeportes.com/efd68/arbitra.htm

13. Guillén, F., Morán, M. y Castro, J. J. (1999). Consideraciones de carácter psicosocial sobre el arbitraje y el juicio deportivo. En F. Guillén (Ed.), La psicología del deporte en España al 


\section{Personalidad y arbitraje de élite del baloncesto español}

final del milenio. VI Congreso Nacional de Psicología de la Actividad Física y el Deporte (pp. 583-591). Las Palmas de Gran Canaria: Universidad de Las Palmas de Gran Canaria.

14. Hill, D. M., Matthews, N. y Senior, R. (2016). The psychological characteristics of performance under pressure in professional rugby union referees. The Sport Psychologist, 30(4). doi:10.1123/tsp.2015-0109

15. Ittenbach, R. F. y Eller, B. F. (1988). A personality profile of Southeastern Conference football officials. Journal of Sport Behavior, 11, 115-125.

16. Johansen, B. T., Haugen, T. (2013). Anxiety level and decision-making among Norwegian top-class soccer referees. International Journal of Sport and Exercise Psychology, 11(2), 215-226. doi:10.1080/1612197X.2013.773665

17. Lirgg, C. D., Feltz, D. L. y Merrie, M. D. (2016). Self-efficacy of sports officials: A critical review of the literature. Journal of Sport Behavior, 39(1), 39-50.

18. Martin, B. R. (2009). Game-related acutely stressful events and coping styles of registered interscholastic sport officials (Tesis doctoral no publicada). Middle Tennessee State University, Murfreesboro, USA.

19. Myers, N. D., Feltz, D. L., Guillén, F. y Dithurbide, L. (2012). Development of, and initial validity evidence for, the Referee SelfEfficacy Scale: A multistudy report. Journal of Sport \& Exercise Psychology, 34(6), 737-765.

20. Myers, T. y Balmer, N. (2012). The impact of crowd noise on officiating in MuayThai: Achieving external validity in an experimental setting. Frontiers in Psychology, 3, Article 346. doi:10.3389/fpsyg.2012.00346

21. Neville, T. J., Salmon, P. M. y Read, G. J. M. (2017). Analysis of in-game communication as an indicator of recognition primed decision making in elite Australian rules football umpires. Journal of Cognitive Engineering and Decision Making, $\quad 11(1), \quad 81-96$. doi:10.1177/1555343416672557

22. Pla-Cortés, J., Gomà-i-Freixanet, M. y AvilésAntón, O. (2015). Perfil de personalidad de los árbitros de baloncesto españoles en comparación con la población general. Cuadernos de Psicología del Deporte, 15(2), 87-94.

23. Sinclair, G. D. (1975). Personality characteristics of nationally rated volleyball officials. Volleyball Technical Journal, 3, 72-80.

24. Souchon, N., Livingstone, A. G., Bardin, B., Rascle, O., Cabagno, G. y Maio, G. R. (2016). Influence of competition level on referees' decision-making in handball. Social Influence, 11(4), 246-258. doi:10.1080/15534510.2016.1259656

25. Spurgeon, J. H., Blair, S. V., Keith, J. A. y McGinn, C. J. (1978). Characteristics of successful and probationary football officials. Physician \& Sportsmedicine, 6, 106-110; 112.

26. Unkelbach, C. y Memmert, D. (2010). Crowd noise as a cue in referee decisions contributes to the home advantage. Journal of Sport \& Exercise Psychology, 32(4), 483-498.

27. Winters, J. C. (2010). Discriminate analysis of the personality characteristics of major college baseball umpires (Tesis doctoral no publicada). Oklahoma State University. Stillwater, Oklahoma, USA.

28. World Medical Association (2013). World Medical Association Declaration of Helsinki: Ethical principles for medical research involving human subjects. JAMA, 310, 2191-2194. http://dx.doi.org/10.1001/jama.2013.281053 